\title{
PREVALENCE OF OPHTHALMIC MANIFESTATIONS IN SPONDYLOARTHRITIS
}

Matheus Diniz Araújo Teixeira1, *, Sandra Lúcia Euzébio Ribeiro1

1.Universidade Federal do Amazonas, Manaus (AM), Brazil.

*Corresponding author: matheus.atdiniz@gmail.com

\section{BACKGROUND}

Spondyloarthritis (SpA) encompasses a group of chronic inflammatory diseases that may have common clinical, genetic and radiological characteristics. The musculoskeletal system is the main site of involvement in SpA and ophthalmic complaints, especially acute anterior uveitis (AAU), represent the most reported extra-articular manifestation. This occurs in greater prevalence in patients with ankylosing spondylitis (AS) and in individuals who are positive for histocompatibility leukocyte antigen B27 (HLA-B27). This work proposes to survey and analyze ophthalmological manifestations in individuals with SpA followed at the rheumatology service.

\section{MATERIALS AND METHODS}

Observational and retrospective research that analyzed patients with SpA who followed a referred rheumatology service from January 2009 to January 2019. This research project was initially submitted to the evaluation and approval of the research Ethics Committee of the Plataforma Brasil and approved under opinion 3.532.081.

\section{RESULTS}

Of the 109 patients evaluated, the mean age was $51.99 \pm 11.29$ years and the mean disease duration was $14.37 \pm 8.73$ years, of which 76 (69.72\%) male gender and 33 (30.27\%) females. Regarding the etiology of the disease, 81 (74.31\%) of AS, 24 (22.02\%) have a diagnosis of psoriatic arthritis (PSA) and four (3.66\%) of undifferentiated arthritis. Histocompatibility leukocyte antigen B27 was positive in $64(58.71 \%)$ and negative in $45(41.29 \%)$ patients with SpA. Of these which were positive for the antigen, $91.66 \%$ have AS and $8.34 \%$ PSA. With regard to ophthalmological manifestations of SpA patients analyzed were found: AAU (29.24\%), pterygium (11.92\%), cataract (9.17\%), glaucoma (6.42\%) and amaurosis $(0.91 \%)$. The presence of HLA-B27 (+) in patients who had AAU $(77.42 \%)$ and glaucoma (80\%) was considerably high. Regarding family history, 23 participants have a diagnosis of SpA in the family, of these, 20 are HLA-B27 positive.

\section{CONCLUSION}

In agreement with literature, the most prevalent ocular manifestation in our study was AUU. It was significantly more frequent in HLA-B27 (+) patients. In daily practice, the determination of HLA-B27 is recommended in all patients with AAU, as it affects the diagnosis and prognosis. In addition, it is worth emphasizing the importance of the Sistema Único de Saúde (SUS) for Brazilian patients with SpA, since SUS has been fully monitoring them in different areas of health for decades. 